\title{
COMPARACIÓN ENTRE EL CLIVAJE TEMPRANO Y EL ESTADIO PRONUCLEAR COMO PARÁMETROS DE REFERENCIA PARA INDUCIR EL EMBARAZO EN PACIENTES OVORECEPTORAS EN CALI (COLOMBIA)
}

\section{Comparing early cleavage and pronuclear state as reference parameters for inducing pregnancy in embryo implantation patients in Calli, Colombia}

María Esther Estela-Paz, Biol*, Jaime Saavedra-Saavedra, M.D.**, Martha Norah Moyano, Biol, MSc.***

Recibido: diciembre 11/08 - Aceptado: agosto 24/09

\section{RESUMEN}

Introducción: algunos autores han propuesto métodos no invasivos que se centran en el clivaje temprano del estadio de dos células a las 25 y 27 horas luego de ocurrida la inseminación, los cuales son aplicables a los programas de fertilización in vitro que aumentan la probabilidad de implantación y embarazo.

Objetivo: comparar la tasa de embarazo en pacientes ovoreceptoras con embriones provenientes de clivaje temprano con respecto a la del estadio pronuclear.

Metodología: se realizó un estudio de cohorte retrospectivo, en el cual se incluyeron a las pacientes ovoreceptoras que asistieron entre abril de 2004 y diciembre de 2007 al Centro de Biomedicina - FECUNDAR en Cali (Colombia) por presentar baja respuesta al ciclo de estimulación ovárica

* Bióloga. Especialista en Embriología Humana, FECUNDAR, Cali (Colombia). Correo electrónico: marespaz@yahoo.com.mx.

** Profesor Titular, Universidad del Valle. Maestro de la especialidad de Ginecología y Obstetricia del Valle del Cauca. Director general de FECUNDAR, Cali (Colombia).

*** Bióloga. MSc en Epidemiología. Docente de la Universidad del Valle. Epidemióloga FECUNDAR, Cali (Colombia). controlada, factor edad, falla ovárica o por ser pacientes subrogadas o gestacionales. El tamaño de la muestra se dividió de la siguiente manera: el porcentaje de embarazo esperado para el grupo de clivaje temprano-singamia (grupo 1) fue 79\% (36 pacientes) mientras que el porcentaje de embarazo para el grupo pronuclear (grupo 2) llegó a ser 21\% (9 pacientes). A partir de la obtención de estos datos, se determinaron las tasas de implantación y de embarazo y se compararon los dos grupos por medio de la prueba Chi-cuadrado $\left(\mathrm{X}^{2}\right)$.

Resultados: de un total de 289 cigotos, se incluyeron 183 que cumplieron los parámetros propuestos, entre embriones provenientes de clivaje temprano (CT), singamia (S) y estadio pronuclear (PN) a las 25 y 27 horas posinseminación. De este total, 79\% provenían del estadio clivaje temprano-singamia y 21\% del estadio pronuclear. El promedio de embriones transferidos a las pacientes ovoreceptoras fue tres embriones, la edad promedio fue 38 años, la tasa de embarazo obtenida al transferir los embriones provenientes de clivaje temprano fue $44 \%$ en comparación con $41 \%$ para la transferencia de los embriones no clivados a 
las 25 horas posinseminación. Finalmente, no se encontraron diferencias significativas en la obtención del embarazo.

Conclusión: bajo las condiciones del estudio, el resultado muestra que no hay diferencias en la tasa de éxitos para ambos procedimientos.

Palabras clave: fertilización, clivaje, ovoreceptoras, singamia, pronuclear, inseminación.

\section{SUMMARY}

Introduction: some authors have proposed that non-invasive embryo implantation methods (based on early cleavage or pronuclear stage for two cells 25 and 27 hours post-insemination) are applicable to in vitro fertilisation programmes, thereby increasing the probability of implantation and pregnancy.

Objective: comparing pregnancy rates in patients implanted with embryos originating from early cleavage to those from the pronuclear stage.

Methodology: a retrospective cohort study was carried out. Embryo implantation patients were included who had been attending the FECUNDAR -Biomedicine Centre in Cali, Colombia, from April 2004 to December 2007 who presented a poor response to the controlled ovarian stimulation cycle, age factor and/or ovarian failure and pregnancy substitute patients. The sample consisted of 45 patients; 36 patients (79\%) were expected to become pregnant in the early-syngamy and cleavage group (group 1) and 9 patients (21\%) in the pronuclear group (group 2). Implantation and pregnancy rates were determined and the Chi-square $\left(\mathrm{X}^{2}\right)$ test was used for comparing both groups.

Results: 183 of the 289 zygotes complied with the proposed parameters (i.e. embryos from early cleavage, syngamy and pronuclear stage 25 and 27 hours post-insemination); 79\% of these 183 came from the early cleavage and syngamy stage and $21 \%$ from the pronuclear stage. Three embryos on average were transferred to embryo implantation patient; average age was 38 , the pregnancy rate obtained when transferring early cleavage embryos was $44 \%$ compared to $41 \%$ for non-cleaved embryo transfer 25 hours post-insemination. No statistically significant differences were found regarding how pregnancy was induced.

Conclusion: equal success rates were obtained for both procedures in the given study conditions.

Key words: fertilisation, cleavage, embryo implantation, syngamy, pronuclear, insemination.

\section{INTRODUCCIÓN}

En reproducción asistida, se utilizan criterios cada vez más estrictos para seleccionar el mejor embrión a transferir con una alta calidad y así evitar una tasa de embarazos múltiples, determinando parámetros de evaluación como lo son el puntaje de los pronúcleos y el clivaje temprano. ${ }^{1}$

Siempre ha existido una inquietud con respecto al número de embriones que se deben transferir para poder proporcionarle a las parejas un resultado positivo en cuanto a embarazo se refiere. A la vez, se evita transferir una cantidad grande de embriones que pueda ocasionar un embarazo múltiple por las consecuencias que ésto conlleva. Por ello, para proporcionar una mejor posibilidad de gestación, varios autores han determinado una sumatoria de parámetros de evaluación desde las 17 hasta las 48 ó 72 horas posrecuperación ovocitaria con el objetivo de seleccionar el mejor embrión a transferir. ${ }^{1-10}$

Algunos investigadores sugieren que transferir en estado pronuclear incrementa la probabilidad de éxito; ${ }^{5}$ sin embargo, los parámetros de calidad embrionaria están relacionados con la edad de la mujer, por lo que se recomienda realizar la evaluación de la eficacia a partir de los óvulos de donantes jóvenes para garantizar así la calidad ovocitaria. Otros factores citados son: la patología de base de la mujer, así como de la calidad seminal, la cual al parecer está implicada con la fragmentación además, esta última puede afectar el éxito del embarazo ya que conlleva a una alta incidencia de desórdenes cromosomales. ${ }^{11}$ Otros autores ${ }^{12}$ sugirieron una observación de 12 a 16 horas pos ICSI (Inyección 
Intracitoplasmática de Espermatozoides) del desarrollo pronuclear para tener un predictor posible de desarrollo preimplantacional.

Se sabe que el primer clivaje predice un desarrollo y una competencia del embrión in vitro a una posible llegada al estadio de blastocisto y posterior preimplantación del mismo, dando como resultado una nueva vida. La probabilidad de alcanzar el estadio de blastocisto cuando el embrión proviene de clivaje temprano (CT), singamia (S) o estado pronuclear (PN) entre las 25 y 27 horas es reportado en 59\%, 52\% y 33\%, respectivamente. ${ }^{13}$ De acuerdo al presente estudio, esta institución quiso verificar si la hipótesis planteada por dichos autores en cuanto a que el primer clivaje es un predictor posible de desarrollo preimplantacional y por ende de la obtención de un embarazo. Por lo tanto, se determinó usar óvulos de donantes jóvenes sanas que garantizaran la calidad del embrión y para poder así comprobar dicha hipótesis.

Por consiguiente, el objetivo de este estudio es comparar la tasa de embarazo en pacientes ovoreceptoras con embriones provenientes de clivaje temprano y singamia (CT y S) con respecto a la de estadio pronuclear (PN).

\section{METODOLOGÍA}

Se realizó un estudio de cohorte retrospectivo, en el cual se incluyeron a todas las pacientes ovoreceptoras que asistieron durante el período de abril de 2004 a diciembre de 2007 al Centro de Biomedicina Reproductiva - FECUNDAR, Cali (Colombia). Ésta es una institución privada de referencia para el estudio y manejo de parejas con infertilidad.

En el estudio se incluyeron las pacientes ovoreceptoras que presentaron los siguientes factores diagnósticos de infertilidad: baja respuesta al ciclo de estimulación ovárica controlada, factor edad, falla ovárica y pacientes subrogadas o gestacionales (pacientes útero-receptor). Como criterios de exclusión se tomaron el factor uterino (endometriosis, miomatosis) y factor masculino (oligozoospermia severa), también las pacientes cuyos embriones transferidos a las 48 ó 72 horas posaspiración ovocitaria provenían de una mezcla de parámetros (clivaje temprano (CT), singamia (S) y estado pronuclear (PN)) a las 25 horas posinseminación.

Tamaño de muestra: con base en un porcentaje esperado de embarazo del grupo de CT y S de $80 \%$ y el porcentaje de embarazo del grupo PN de 25\%, un poder de $80 \%$, un intervalo de confianza de $95 \%$ y una razón de 4:1, se estimó un tamaño de muestra de 36 pacientes en el grupo CT y S, y de 9 pacientes en el grupo PN para un total de 45 pacientes. Se sumó $10 \%$ asumiendo alguna pérdida, con lo cual el resultado fue de 50 pacientes. Finalmente, se llevó a cabo un muestreo secuencial consecutivo.

Procedimiento: previo al procedimiento de fertilización in vitro (IVF), las pacientes donantes recibieron el agente bloqueante: acetato de leuprolide (Lupron ${ }^{\circledR}$, Abbott, Francia) en una dosis de 0,5 mg/día. El día 2 ó 3 del ciclo se inició la aplicación de la hormona folículo estimulante (FSH, por sus siglas en inglés) en dosis de 150 a 225 UI por un período de 5 días y luego se continuó con la hormona gonadotropina menopáusica humana (HMG, por sus siglas en inglés) en las mismas dosis. En el día 5 de la estimulación ovárica, se inició la monitorización del desarrollo folicular por ecografía vaginal; las dosis de gonadotropinas se adaptaron de acuerdo con la respuesta ovárica. Cuando el mayor de los folículos medía $20 \mathrm{~mm}$, se suministró la gonadotropina coriónica humana (HCG) en una dosis de 10.000 UI. Después de 34 a 38 horas de la aplicación del HCG se procedió a la aspiración de los folículos a través de una punción transvaginal guiada por ultrasonido. Una vez identificados los ovocitos en el fluido folicular, éstos fueron clasificados por su madurez y se dejaron en la incubadora en medio de cultivo cuyos componentes son: sales minerales, aminoácidos, vitaminas y antibióticos a $5 \%$ de $\mathrm{CO}_{2}$ y $37^{\circ} \mathrm{C}$. La preparación endometrial de las pacientes ovoreceptoras fue realizada con valerato de estradiol (progynova $^{\circledR}$ ) $2 \mathrm{mg} /$ día iniciando el día 2 del ciclo y acetato de leuprolide (Lupron ${ }^{\circledR}$, Abbott, Francia) 
aplicado desde el día 21 del ciclo previo; en pacientes menopáusicas solamente se usó valerato de estradiol (progynova $^{\circledR}$ ) 2 mg/día. En ambos casos, se aumentó la dosis de valerato de estradiol (progynova ${ }^{\circledR}$ ) de acuerdo al desarrollo del endometrio, el cual fue medido por valoración ecográfica intravaginal. Se consideró un endometrio apto para transferencia embrionaria cuando tenía un grosor de 8 - $12 \mathrm{~mm}$ con patrón trilaminar

Transcurridas 4 a 7 horas posaspiración folicular, los ovocitos fueron inseminados con 50.000, 100.000 ó 150.000 espermatozoides de acuerdo a la calidad espermática, en micro gotas de $50 \mu \mathrm{l}$ de medio de cultivo bajo aceite mineral. Después de 17 a 19 horas posinseminación, los ovocitos fueron denudados y observados en microscopio invertido para determinar la presencia o ausencia de pronúcleos (fertilización), ${ }^{14}$ los cuales se evaluaron según el criterio estándar. ${ }^{2,9,15-17}$ Posteriormente, 25 a 27 horas posinseminación se evaluó el CT (clivaje temprano) o S (singamia) o PN (pronúcleos), al siguiente día se observó y analizó el segundo y tercer clivaje (4 y 8 células, respectivamente), y se realizó la selección de embriones a transferir. Se determinó realizar dos grupos de estudio basados en investigaciones previas, ${ }^{1-10,13}$ en los cuales se comprobó que el clivaje temprano es un fuerte indicador de la calidad del embrión humano y la probabilidad de quedar en embarazo.

El grupo I correspondió a las pacientes cuya transferencia de embriones se realizó a las 48 a 72 horas posinseminación provenientes de CT y $\mathrm{S}$ (45 ovoreceptoras) y el grupo II correspondió a las pacientes cuya transferencia fue realizada a las 48 a 72 horas posinseminación provenientes del estadio PN (12 ovoreceptoras).

En todos los casos, los embriones que presentaron CT, Sy PN fueron transferidos cuando no presentaban más de $20 \%$ de fragmentación. Los embriones fueron seleccionados de acuerdo a su calidad (grado I, grado II). Los grado I son embriones con blastómeras simétricas, cada una con su núcleo, sin fragmentación y citoplasma homogéneo mientras que los grado II son embriones con blastómeras simétricas, cada una con su núcleo, con 5\% a 10\% de fragmentación y citoplasma homogéneo. Los catéteres utilizados para las transferencias fueron Frydman TD ref. 1303000 y Frydman ultrasoft ref. 1324301.

La prueba de embarazo químico fue realizada en el día 16 después de la transferencia, determinando la presencia de la hormona beta gonadotropina coriónica humana (B-HCG); la prueba se consideró positiva cuando el resultado fue mayor de 20 IU y el embarazo clínico fue confirmado en la tercera semana por la presencia del saco gestacional y embriocardia positiva mediante ecografía.

Separación por gradientes de densidad: para la separación de los espermatozoides del fluido seminal se utilizaron los líquidos de alta densidad como el Isolate (Irvine Scientific ${ }^{\circledR}$ ), en gradientes de $90 \%$ y 40\%, y los parámetros de evaluación seminal fueron determinados de acuerdo con los criterios de la OMS (Organización Mundial de la Salud). Dependiendo de la concentración espermática de la muestra, se varió el volumen de cada gradiente; para una muestra espermática mayor de 40 millones $/ \mathrm{mL}$ se realizaron gradientes de 1,5 mL, para una de $30-10$ millones/mL, gradientes de 1,0 mL y por debajo de 10 millones $/ \mathrm{mL}$, gradientes de 0,5 mL de 90\% y 40\%, respectivamente. Después de realizar lo anterior, se realizó la inseminación de los óvulos (50.000 - 150.000 espermatozoides/óvulo).

Para el análisis estadístico, se obtuvo la base de datos en Excel $^{\circledR}$, la cual se analizó para su procesamiento. Posteriormente, se transfirió dicha base de datos al programa estadístico Stata $6.0^{\circledR}$. Las variables continuas se analizaron por medio de las medidas de tendencia central, y de las variables categóricas se obtuvieron las proporciones. Inmediatamente después, se compararon las características de las pacientes así como la obtención del embarazo con respecto a los procedimientos de CT - S y PN, mediante pruebas de $\mathrm{X}^{2}$ (Chi-cuadrado) a un nivel de significancia de $95 \%$. 


\section{RESULTADOS}

Durante el período de estudio se incluyeron 45 pacientes en el grupo I y 12 pacientes en el grupo II, para un total de 289 cigotos que presentaban un estadio embrionario entre las $25 \mathrm{y}$ 27 horas posinseminación, de los cuales fueron incluidos sólo 183. Se transfirieron los embriones, de los cuales 79\% (144/183) estaban en el estadio de CT - S (grupo I) y 21\% (39/183) provenientes del estadio de PN (grupo II). Finalmente, se seleccionaron 57 pacientes ovoreceptoras divididas en dos grupos de acuerdo al desarrollo o no de CT. Los 109 cigotos restantes no se incluyeron en el estudio porque las pacientes ovoreceptoras fueron transferidas con embriones provenientes de todos los estadios (CT - S y PN).

La edad promedio de las pacientes fue 38 años, la menor edad encontrada fue 18 y la mayor 47 años presentándose una mayor frecuencia en las edades comprendidas entre 35 y 45 años (75\%).

El promedio de embriones transferidos fue 3,2 (56,2\% de pacientes ovoreceptoras fueron transferidas con tres embriones, 33,3\% con 4 embriones, $8,8 \%$ con 2 embriones y 1,7\% con un embrión). Con respecto al promedio del grosor endometrial (trilaminar), $10 \mathrm{~mm}$ fue la medida más constante. De este modo, el menor desarrollo endometrial encontrado fue $6 \mathrm{~mm}$ en 2 pacientes (3,5\%) pero también se encontró un desarrollo de $8 \mathrm{~mm}$ en 4 pacientes $(7,0 \%)$, de $9 \mathrm{~mm}$ en 16 pacientes $(28,1)$ y de $10 \mathrm{~mm}$ en 13 pacientes (22,8\%). Asimismo, 4 pacientes presentaron $11 \mathrm{~mm}$ (24,6\%); 7 pacientes, $12 \mathrm{~mm}(12,3 \%)$ y una paciente, $13 \mathrm{~mm}$.

La tabla 1 representa las frecuencias de los diagnósticos de las 57 pacientes ovoreceptoras. El diagnóstico que presentó mayor frecuencia es el factor edad, seguido de los factores falla ovárica y pacientes subrogadas o gestacionales, los cuales presentan una tasa de embarazo de 55,5\%, 15,3\% y 50\%, respectivamente.

De otra parte, las características basales de las pacientes ovoreceptoras se presentan en la tabla 2. Los dos grupos no presentan diferencias clínica ni
Tabla 1. Frecuencia de diagnósticos en las pacientes

ovoreceptoras transferidas en FECUNDAR desde abril 2004 a diciembre 2007.

\begin{tabular}{|l|c|c|}
\hline Diagnóstico & $\begin{array}{c}\text { Frecuencia } \\
(\mathbf{n})\end{array}$ & $\begin{array}{c}\text { Porcentaje } \\
\%\end{array}$ \\
\hline Factor edad & 27 & 47,5 \\
\hline Falla ovárica & 13 & 22,8 \\
\hline Baja respondedora & 7 & 12,1 \\
\hline Subrogada o gestacional & 10 & 17,6 \\
\hline TOTAL & 57 & 100,0 \\
\hline
\end{tabular}

Fuente: base de datos FECUNDAR.

estadísticamente significativas. Además, la tasa de embarazo de embriones clivados en comparación con embriones no clivados a las 25 a 27 horas posinseminación fue de 44,4\% vs. 41,7\% ( $\mathrm{p}=0,863)$.

Por otra parte la tasa de embarazo de embriones clivados en comparación con embriones no clivados a las 25 a 27 horas posinseminación no presentó una diferencia estadísticamente significativa $p=0,863$, (44,4\% vs. 41,7\%). Tabla 2. Tampoco se encontraron diferencias significativas en el promedio de edad, promedio de embriones transferidos y promedio de grosor endometrial en las pacientes ovoreceptoras transferidas con embriones provenientes de CT y S comparado con las pacientes ovoreceptoras transferidas con embriones provenientes de PN. Tabla 2.

\section{DISCUSIÓN}

La selección de las pacientes ovoreceptoras según las características que presentaron a las 25 a 27 horas posinseminación mostró que el origen de los gametos (óvulos y espermatozoides) son una base importante en la obtención de embriones que cumplieron con los parámetros propuestos para este estudio: no obstante, no se encontraron diferencias estadísticamente significativas en cuanto a la obtención de embarazo entre los grupos estudiados (de las 57 pacientes ovoreceptoras seleccionadas, 44\% de las pacientes logró un embarazó bajo estos criterios). 
Los resultados no son consistentes con los estudios que reportan que hay diferencias significativas entre los grupos con clivaje temprano con respecto a los de estadio pronuclear como una herramienta de pronóstico del embarazo para pacientes que acuden a técnicas de reproducción asistida., ${ }^{1,3,710}$ Además, el estudio pudo evidenciar que aumentando el número de embriones provenientes de CT en la transferencia del grupo I, éste tiene una tendencia a incrementar la tasa de gestaciones múltiples. Ésto se apoya en estudios previos que muestran un marcado incremento de las tasas de embarazo ${ }^{9}$ y embarazo múltiple ${ }^{3}$ cuando el grupo de transferencias incluye tres embriones de buena calidad en lugar de uno o dos. Con respecto al porcentaje de aborto, tampoco se observaron diferencias significativas entre los dos grupos de estudio (CT - S vs. estadio PN), lo cual está de acuerdo con en el estudio realizado por Aafke y colaboradores, ${ }^{10}$ quienes encontraron un incremento de aborto en el grupo de pacientes transferidas con embriones que no hicieron clivaje temprano, comparado con el grupo de los que clivaron tempranamente. Ahora bien, aunque la diferencia no es estadísticamente significativa, ésto es similar a lo que encontraron Lundin y su equipo de trabajo ${ }^{4}$ cuando se dedicaron a analizar un gran número de ciclos.

Con el presente estudio se esperaba obtener una diferencia en la proporción de embarazo de 55\% entre los grupos a comparar; sin embargo, los resultados indican que no hay evidencia de una diferencia en obtención de embarazo en ambos grupos de estudio con respecto al desarrollo embrionario a las 25 horas posinseminación. La ausencia de diferencia puede ser el resultado de una subestimación del tamaño muestral por lo que se recomienda realizar un estudio con un tamaño de muestra mayor para verificar así los hallazgos del estudio.

Además, se recomienda, como estudio complementario, evaluar la tasa de embarazo en los grupos de clivaje temprano - singamia o estadio pronuclear categorizados. Aún así, esta investigación es el resultado de procedimientos técnicos estandarizados lo cual garantiza la validez de los datos.

En conclusión, el presente estudio mostró que independientemente de que los cigotos hayan clivado o no a las 25 a 27 horas posinseminación, no hay diferencia significativa en cuanto a la obtención del embarazo en pacientes ovoreceptoras que recibieron embriones provenientes de estos estadios (clivaje

Tabla 2. Características de transferencia de embriones en pacientes con CT y S en comparación con embriones que no tuvieron clivaje temprano para pacientes ovoreceptoras abril de 2004 a diciembre de 2007 - FECUNDAR.

\begin{tabular}{|c|c|c|c|}
\hline Características & $\begin{array}{l}\text { Clivaje temprano } \\
\text { y singamia } \\
\text { Grupo I } \\
\text { n=45 }\end{array}$ & $\begin{array}{l}\text { Pronúcleos Grupo II } \\
n=12\end{array}$ & Valor $p$ \\
\hline Edad (promedio) & $38(\mathrm{DS}= \pm 6,64)$ & $38(\mathrm{DS}= \pm 4,76)$ & 0,6783 \\
\hline $\begin{array}{l}\text { Número de embriones transferidos } \\
\text { (Promedio) }\end{array}$ & $3,2(\mathrm{DS}= \pm 0,72)$ & $3,2(\mathrm{DS}= \pm 0,45)$ & 0,8219 \\
\hline $\begin{array}{l}\text { Desarrollo endometrial } \\
\text { (Promedio-mm) }\end{array}$ & $9,9(\mathrm{DS}= \pm 1,49)$ & $10,3(\mathrm{DS}= \pm 1,21)$ & 0,4716 \\
\hline Tasa de aborto (\%) & 0 & 4,44 & 0,457 \\
\hline Tasa de embarazo (\%) & 44,4 & 41,7 & 0,863 \\
\hline Tasa de embarazo múltiple (\%) & 36 & 0 & 0,090 \\
\hline
\end{tabular}

Fuente: base de datos FECUNDAR. 
temprano-singamia y pronuclear), lo cual indica que el resultado es exitoso para los dos grupos de estudio.

\section{AGRADECIMIENTOS}

Los autores desean agradecer a todo el personal médico de embriología y de administración del Centro de Biomedicina Reproductiva - FECUNDAR quienes de una u otra forma aportaron sus valiosos conocimientos para realizar el presente estudio.

\section{REFERENCIAS}

1. Bos-Mikich A, Mattos AL, Ferrari AN. Early cleavage of human embryos: an effective method for predicting successful IVF/ICSI outcome. Hum Reprod 2001;16:2658-61.

2. Edwards RG, Steptoe PC, Purdy JM. Establishing fullterm human pregnancies using cleaving embryos grow in vitro. Br J Obstet Gynaecol 1980;87:737-56.

3. Fenwick J, Platteau P, Murdoch AP, Herbert M. Time from insemination to first cleavage predicts developmental competence of human preimplantation embryos in vitro. Hum Reprod 2002;17:407-12.

4. Lundin K, Bergh C, Hardarson T. Early embryo cleavage is a strong indicator of embryo quality in human IVF. Hum Reprod 2001;16:2652-7.

5. Ahuja K, Smith W, Tucker M, Craff I. Successful pregnancies from the transfer of pronucleate embryos in an outpatient in vitro fertilization program. Fertil Steril 1985;4:181-4.

6. Salumets A, Hyden-Granskog C, Mäkinen S, Suikkari AM, Titinen A, Tuuri T. Early cleavage predicts the viability of human embryos in elective single embryo transfer procedures. Hum Reprod 2003;18:821-5.

7. Sakkas D, Shoukir Y, Chardonnens D, Bianchui PG, Campana A. Early cleavage of human embryos to the two cell stage after intracitoplasmic sperm injection as an indicator of embryo viability. Hum Reprod 1998;13:182-7.
8. Shoukir Y, Campana A Frealey T, Sakkas D. Early cleavage of in vitro fertilized embryos to the 2-cell stage: a novel indicator of embryo quality and viability. Hum Reprod 1997;12:1531-6.

9. Ziebe S, Petersen K, Lindenberg S, Andersen AG, Gabrielsen A, Andersen AN. Embryos morphology oar cleavage stage: how to select the best embryos for transfer after in vitro fertilization. Hum Reprod 1997; $12: 1545-9$.

10. Van Montfoort AP, Dumoulin JC, Kester AD, Evers JL. Early cleavage is a valuable addition to existing embryo selection parameters: a study using single embryo transfers. Hum Reprod 2004;19:2103-8.

11. Salumets A, Suikkari AM, Möls T, Söderström-Anttila $\mathrm{V}$, Tuuri T. Influence of oocytes and spermatozoa on early embryonic development. Fertil Steril 2002;78:1082-7.

12. Rienzi L, Ubaldi F, Iacobelli M, Ferrero S, Minasi MG, Martinez F, et al. Day 3 embryo transfer with combined evaluation at the pronuclear and cleavage stages compares favourably with day 5 blastocyst transfer. Hum Reprod 2002;17:1852-5.

13. Wiemer KE. Early cleaveage as well as syngamy are positive pronosticator of subsequent human embryonic development. Fertility World 2004;1:11.

14. Scott LA, Smith S. The successful use of pronuclear embryo transfers the day following oocyte retrieval. Hum Reprod 1998;13:1003-13.

15. Alikani M, Calderon G, Tomkin G, Garrisi J, Kokot M, Cohen J. Cleavage anomalies in early human embryos and survival after prolonged culture in vitro. Hum Reprod 2000:15:2634-43.

16. Hurts BS, Tucker KE, Awoniyi CA, Schlaff WD. Pronuclear uterine transfer lowers in vitro fertilization success. Assist Reprod Genet 1998;15:575-7.

17. Scott LA, Alvero R, Leondires M, Miller B. The morphology of human pronuclear embryo is positively related to blastocyst development and implantation. Hum Reprod 2000;15:2394-403. 